\title{
On the use of the average large separation in surface layer independent model fitting and mass estimation (Research Note)
}

\begin{abstract}
Ian W. Roxburgh
Astronomy Unit, Queen Mary University of London, Mile End Road, London E1 4NS, UK

e-mail: I.W.Roxburgh@qmul.ac.uk

Received 8 June 2014 / Accepted 1 September 2014

ABSTRACT

The physics of the outer layers of a star are not well understood but these layers make a major contribution to the large separation. We quantify this using stellar models and show that the contribution ranges from $6 \%$ from the outer $0.1 \%$ of the radius to $30 \%$ from the outer 5\%; it would therefore be inconsistent to impose the large separation as a constraint on surface layer independent model fitting. The mass and luminosity are independent of the outer layers and can be used as constraints, the mass being determined from binarity or from surface gravity and radius. The radius can be used as a constraint but with enhanced error estimates. Using stellar models we show that the errors in estimating mass from the scaling relation between mass, radius and large separation can be up to $30 \%$, and that the errors are not reduced on using the asymptotic value of the large separation estimated by extrapolation to high frequencies.
\end{abstract}

Key words. stars: oscillations - asteroseismology - stars: interiors - methods: analytical - methods: numerical

\section{Introduction}

The average large separation $\Delta$ of a set of frequencies $v_{n \ell}$ defined as

$\Delta=\left\langle v_{n+1, \ell}-v_{n, \ell}\right\rangle$

is widely used when seeking to infer the properties of an observed star, where $\langle\ldots\rangle$ is some averaging procedure. There is no unanimity on the averaging procedure which may be over all observed frequencies, over a subset of those frequencies around the peak of oscillation power $v_{\max }$, or by the autocorrelation of the observed time series; the values obtained depend on the averaging procedure but are usually within $1 \%$ of each other.

The average large separation is frequently used in model fitting where one seeks a model whose frequencies, or combinations of frequencies, match those of the observed star. There are two approaches to model fitting: one is seek to match the frequencies (possibly incorporating a surface offset derived by a linear scaling of the solar value, Kjeldsen et al. 2008); the second recognises that our modelling of the outer layers of a star is subject to many uncertainties (cf. Christensen-Dalsgaard et al. 1988; Dziembowski et al. 1988) and therefore seeks to match functions of the frequencies which are (almost) independent of the structure of the outer layers of a star, the most popular being the ratio of small to large separations (Roxburgh \& Vorontsov 2003, 2013).

When implementing model fitting procedures it is usual to impose some global constraints on the models such as mass, luminosity, effective temperature, surface gravity, radius, composition, and the average value of the large separation $\Delta$. In Sects. 2 and 3 below we show that the outer layers of a star make a major contribution to the value of the large separation so that, since the objective of surface-layer independent model fitting is to subtract out the effect of the outer layers of the star, it is inconsistent to constrain surface layer independent model fitting by requiring that the model has the observed large separation.

A second use of the average large separation $\Delta$ is to obtain an estimate of the mass $M$ of a star from $\Delta$ and its radius $R$ using a scaling relation which we write in the form

$\frac{M}{M_{\odot}}=\lambda\left(\frac{\Delta}{\Delta_{\odot}}\right)^{2}\left(\frac{R}{R_{\odot}}\right)^{3}$,

where $\Delta_{\odot}(\approx 135 \mu \mathrm{Hz})$ is the solar value (cf. Huber et al. 2011). It is customary to take $\lambda=1$ but in general this is not valid as it depends on the dimensionless structure of the star and is the source of the error in using the relation with $\lambda=1$. It has been argued by Mosser et al. (2013) that were one to extrapolate from the observed $\Delta$ to its asymptotic value $\Delta_{T}=1 /(2 T), T$ being the acoustic radius, then one can use this relation with $\lambda=1$. Quite apart from the difficulties in such an extrapolation (cf. Hekker et al. 2013), we show in Sect. 5 that the uncertainties on using $\Delta_{T}$ are as large as those from using the observed value of $\Delta$.

\section{The contribution of the outer layers to the large separation - simple analysis}

We first consider a simplified analysis based on the first order asymptotic approximation where the oscillation frequencies satisfy the equation (Vandakurov 1967; Tassoul 1980)

$v_{n, \ell}=\Delta_{T}(n+\ell / 2+\epsilon)$, where $\Delta_{T}=\frac{1}{2 T}$ and $T=\int_{0}^{R_{\mathrm{a}}} \frac{\mathrm{d} r}{c(r)}$

is the acoustic radius of the star, $R_{\mathrm{a}}$ is the radius of the top of the atmosphere, $c$ the sound speed and $\epsilon$ is a constant. Since $c(r)$ is smallest in the outer layers the contribution of these layers to $T$ and hence to $\Delta_{T}$ is significant, uncertainties in the structure of the outer layers producing significant uncertainty in $\Delta_{T}$. 
Table 1. Contribution $\delta \Delta$ to $\Delta_{T}$ versus $x_{f}\left(M=1.10 M_{\odot}\right)$.

\begin{tabular}{crrrrrrrr}
\hline \hline$x_{f}$ & 0.0 & 0.50 & 0.90 & 0.95 & 0.97 & 0.99 & 0.995 & 0.999 \\
$\delta \Delta(\mu \mathrm{Hz})$ & 119.6 & 91.16 & 45.4 & 33.5 & 26.4 & 15.7 & 10.7 & 4.4 \\
\hline
\end{tabular}

To quantify this we separate $T$ into its contribution $t_{f}$ from the interior of the star below the radial distance $r_{f}$, and $\tau_{f}$ the contribution from the outer layers above $r_{f}$ :

$$
T=t_{f}+\tau_{f}, \quad t_{f}=\int_{0}^{r_{f}} \frac{\mathrm{d} r}{c(r)}, \quad \tau_{f}=\int_{r_{f}}^{R_{\mathrm{a}}} \frac{\mathrm{d} r}{c(r)} .
$$

On multiplying the definition of $\Delta_{T}$ in Eq. (3) by $2 T \Delta_{T}$ we have

$$
\Delta_{T}=2\left(t_{f}+\tau_{f}\right) \Delta_{T}^{2},
$$

so the contribution to $\Delta_{T}$ from the outer layers above the fractional radius $x_{f}=r_{f} / R$ is $\delta \Delta=2 \tau_{f} \Delta_{T}^{2}$.

In Table 1 we show the contribution $\delta \Delta$ (in $\mu \mathrm{Hz}$ ) from the layers above $x_{f}$ for a model main sequence star (Model A) of $1.10 M_{\odot}$, initial composition $X=0.72, Z=0.02$ evolved to a central hydrogen abundance $X_{\mathrm{c}}=0.25$; with the top of the atmosphere at an optical depth of $10^{-3}$ this has an acoustic radius of $t=4181 \mathrm{~s}$ and hence a $\Delta_{T}=119.6 \mu \mathrm{Hz}$. As can be seen from Table 1 , just the outer $0.1 \%$ of the star contributes $4 \%$ to $\Delta_{T}$ and the outer $5 \%$ contributes $30 \%$, so errors in modelling the outer layers can produce a substantial change in $\Delta_{T}$, much greater than the error estimates on the mean large separation of typical frequency sets obtained by the CoRoT or Kepler missions which are typically $0.1-0.4 \mu \mathrm{Hz}$ (cf. Creevey et al. 2013).

\section{Full analysis}

The first order asymptotic relation (Eq. (2)) is, in general, a poor approximation and the mean large separation $\Delta$ around the peak of $p$-mode power, $v_{\max }$, differs from the value given by the acoustic radius $\Delta_{T}$. For the $1.10 M_{\odot}$ stellar model $\Delta \approx$ $117.0 \mu \mathrm{Hz}$, whereas $\Delta_{T}=119.6 \mu \mathrm{Hz}$. We therefore use a full non-asymptotic analysis to further study the effect of uncertainties in the outer layers, replacing the asymptotic relation (3) by the expression

$v_{n, \ell}=\Delta\left(n+\ell / 2+\epsilon_{n \ell}\right)$,

where $\Delta$ is now an average large separation around $v_{\max }$ and the phase shifts $\epsilon_{n \ell}\left(v_{n, \ell}\right)$ are defined by this relation once the value of $\Delta$ has been specified.

As shown by Roxburgh \& Vorontsov (2000, 2003), Roxburgh (2009), on matching the solution of the oscillation equations integrated away from the centre with the solution integrated in from the surface at any intermediate acoustic radius $t$, the eigenfrequencies of a star satisfy the equation

$$
2 \pi T v=\pi[n+\ell / 2]+\alpha_{\ell}(v, t)-\delta_{\ell}(v, t),
$$

where again $T$ is the total acoustic radius star (from the centre to the top of the atmosphere $r=R_{\mathrm{a}}$ ). This equation is identical in form to Eq. (6), but with $\Delta=1 /(2 T)$ and $\epsilon_{n \ell}=\left(\alpha_{\ell}-\delta_{\ell}\right) / \pi$.

Here $\delta_{\ell}(v, t), \alpha_{\ell}(v, t)$ are inner and outer phase shifts defined by the equations

$$
\begin{aligned}
& \frac{2 \pi v \psi}{\mathrm{d} \psi / \mathrm{d} t}=\tan \left[2 \pi v t-\ell \pi / 2+\delta_{\ell}(v, t)\right] \quad t \leq t_{f}, \\
& \frac{2 \pi v \psi}{\mathrm{d} \psi / \mathrm{d} \tau}=\tan \left[2 \pi v \tau-\alpha_{\ell}(v, \tau]\right] \quad t \geq t_{f},
\end{aligned}
$$

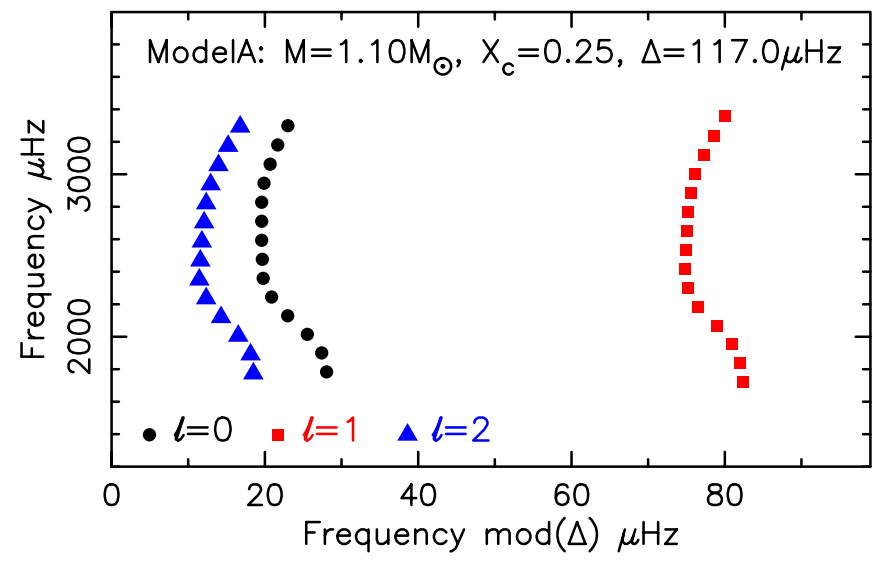

Fig. 1. Echelle diagram of the frequencies of model A, a main sequence star of mass $1.10 M_{\odot}$ with central hydrogen abundance $X_{\mathrm{c}}=0.25$.

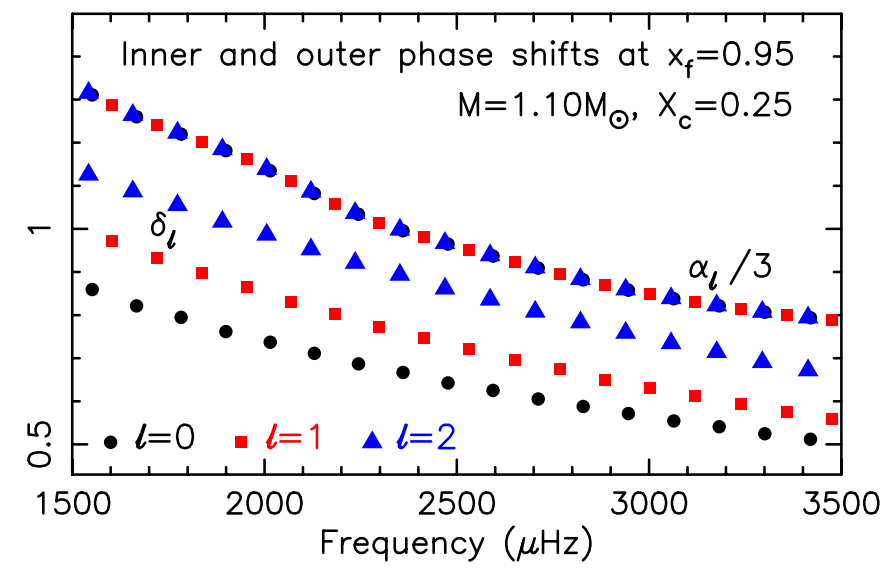

Fig. 2. Phase shifts $\delta_{\ell}(v)$ and $\alpha_{\ell}(v)$ for Model A and modes of degree $\ell=0,1,2$. Note that the $\alpha_{\ell}(v)$ all lie on the same curve.

where $\psi=r p^{\prime} /(\rho c)^{1 / 2}$ with $p^{\prime}(r)$ the Eulerian pressure perturbation, $t$ the acoustic radius at $r$, and $\tau=T-t$ the acoustic depth. For modes of degree $\ell=0,1$, where the fourth order system of oscillation equation collapse to second order (cf. Takata 2005; Roxburgh 2006, 2008a), the $\alpha_{\ell}\left(v, \tau_{f}\right)$ at any acoustic radius $t_{f}$ are determined solely by the structure of the layers above $t_{f}$, and $\delta_{\ell}\left(v, t_{f}\right)$ at any $t_{f}$ are determined solely by the structure interior to $t_{f}$. This is also a very good approximation for modes of degree $\ell=2,3$ provided $t_{f}$ is taken in the outer layers where the density is small.

To demonstrate this we again use the model of a $1.10 M_{\odot}$ main sequence star (ModelA), whose frequencies for $\ell=0,1,2$ are shown in Fig. 1 in a traditional echelle diagram. Figure 2 shows the $\alpha_{\ell}(v)$ and $\delta_{\ell}(v)$ for this model at a fitting radius $t_{f}$, corresponding to a fractional radius $x_{f}=r_{f} / R=0.95$. The result that, to high accuracy, all the $\alpha_{\ell}(v)$ lie on the same curve independent of $\ell$ is the basis of surface layer independent model fitting techniques such as comparing separation ratios.

The phase shifts $\alpha_{\ell}(v, t), \delta_{\ell}(v, t)$ can be evaluated for any acoustic radius $t_{f}$ and any frequency $v$, but for an eigenfrequency $\psi$ and $\mathrm{d} \psi / \mathrm{d} t$ must be continuous. On equating Eqs. (8a) and (8b) at $t_{f}$ we obtain (as in Eq. (7))

$2 \pi v_{n \ell}\left(t_{f}+\tau_{f}\right)-\ell \pi / 2+\delta_{\ell}\left(v_{n \ell}, t_{f}\right)-\alpha_{\ell}\left(v_{n \ell}, t_{f}\right)=n \pi$,

where we have used the trivial result that $A+B=n \pi$ when $\tan (A+B)=0$, for integer $n$. 


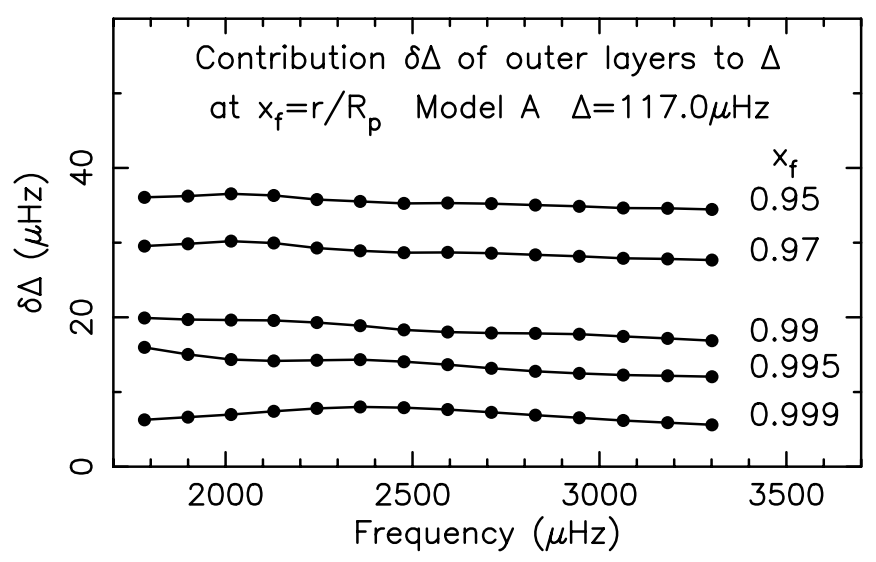

Fig. 3. Contribution $\delta \Delta$ of the outer layers to the large separations $\Delta_{n 0}$ for different depths, for a stellar model of $1.10 M_{\odot}$ with $\Delta \sim 117 \mu \mathrm{Hz}$. $R_{\mathrm{p}}$ is the photospheric radius.

Subtracting this equation from the corresponding equation for $n+1$ we obtain after some manipulation

$\frac{1}{\Delta_{n \ell}}=\left[\left(2 t_{f}+\frac{1}{\pi} \frac{\partial \delta_{\ell}}{\partial v}\right)_{i}+\left(2 \tau_{f}-\frac{1}{\pi} \frac{\partial \alpha_{\ell}}{\partial v}\right)_{o}\right]$,

where

$$
\frac{\partial \delta_{\ell}}{\partial v} \equiv \frac{\delta_{\ell}\left(v_{n+1, \ell}\right)-\delta_{\ell}\left(v_{n, \ell}\right)}{v_{n+1, \ell}-v_{n, \ell}} \quad \frac{\partial \alpha_{\ell}}{\partial v} \equiv \frac{\alpha_{\ell}\left(v_{n+1, \ell}\right)-\alpha_{\ell}\left(v_{n, \ell}\right)}{v_{n+1, \ell}-v_{n, \ell}} .
$$

The first term in brackets with subscript $i$ in Eq. (10) is determined solely by the structure interior to the fitting point $t_{f}$ and the second term with subscript $o$ is determined solely by the structure exterior to $t_{f}$. On multiplying Eq. (10) by $\Delta_{n \ell}^{2}$ we deduce that the contribution of the outer layers to $\Delta_{n \ell}$ is

$\delta \Delta_{n \ell}\left(t_{f}\right)=\left(2 \tau_{f}-\frac{1}{\pi} \frac{\partial \alpha_{\ell}}{\partial v}\right) \Delta_{n \ell}^{2}$.

Figure 3 shows $\delta \Delta$ for modes $\ell=0$ and $n=14,27$, and for different depths, in terms of the fractional radii $x_{f}=r / R_{\mathrm{p}}=$ $0.95,0.97,0.99,0.995,0.999$. Here the outer $0.1 \%$ of the radius contributes $7 \%$ to $\Delta$ and the outer $5 \%$ contributes $30 \%$, in broad agreement with the results of the simple analysis in Sect. 2. So inaccuracies in the modelling of the outer layers can have a large effect on the value of $\Delta$.

These results apply equally to more evolved stars. To show this we undertook the same analysis for Model B, a highly evolved post main sequence star of mass $1.15 M_{\odot}$ with initial composition $X=0.72, Z=0.015$ in the shell burning phase moving over to the red giant branch. The model has many mixed modes as can be seen in the echelle diagram (Fig. 4). The phase shifts are shown in Fig. 5: the inner phase shifts $\delta_{n \ell}$ for $\ell=1,2$ no longer lie on smooth curves but the outer phase shifts $\alpha_{n \ell}$ still all lie on a single curve for all $\ell$. In Fig. 6 we give the contribution of the outer layers to the large separation $\Delta$ for $14 \ell=0$ modes with $n=9,22$, as a function of fractional radius $x_{f}$. Here the outer $0.1 \%$ of the radius contributes $6.5 \%$ to $\Delta$ and the outer $5 \%$ some $32 \%$, more or less the same as for Model A.

\section{Global constraints on surface layer independent model fitting}

The above analysis demonstrates that the even small differences in the structure of the outer layers of a star can make a significant

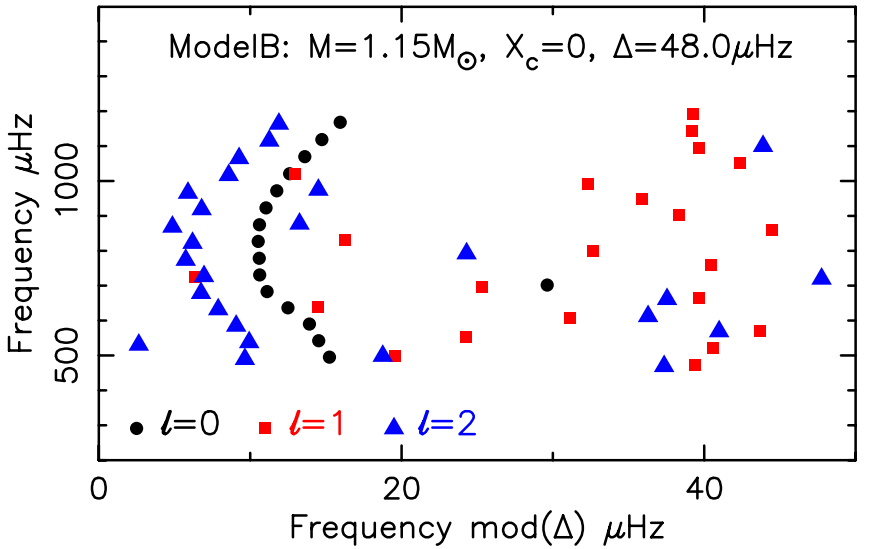

Fig. 4. Echelle diagram of the frequencies of model B, a $1.15 M_{\odot}$ post main sequence model in the shell burning phase - the model has many mixed modes for both $\ell=1$ and $\ell=2$.

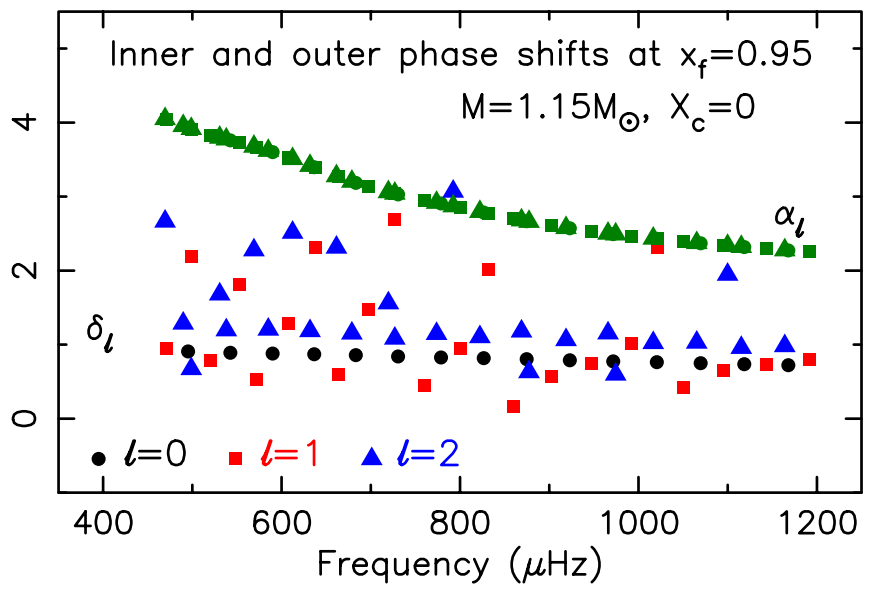

Fig. 5. Inner phase shifts $\delta_{\ell}(v)$ and outer phase shifts $\alpha_{\ell}(v)$ for Model B and modes of degree $\ell=0,1,2$. Note that the $\alpha_{\ell}(v)$ (in green) still all lie on a single curve.

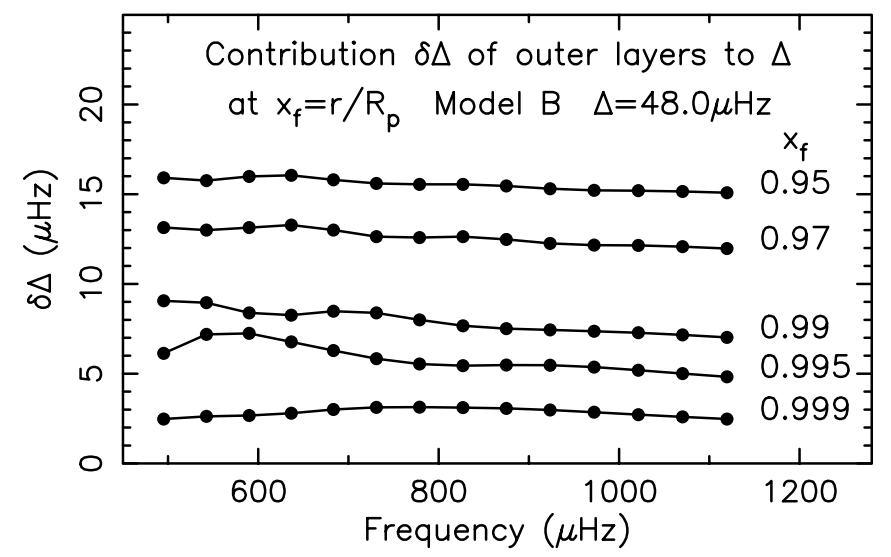

Fig. 6. Contribution $\delta \Delta$ of the outer layers to the large separations $\Delta_{n 0}$ for different depths, for Model B with $\Delta \sim 48 \mu \mathrm{Hz} . R_{\mathrm{p}}$ is the photospheric radius.

difference to the value of the large separation $\Delta$. When using surface layer independent model fitting (separation ratios or phase matching), one is seeking to subtract out the effect of the outer layers so it is inconsistent to constrain the search by requiring the model fit the observed large separation. 


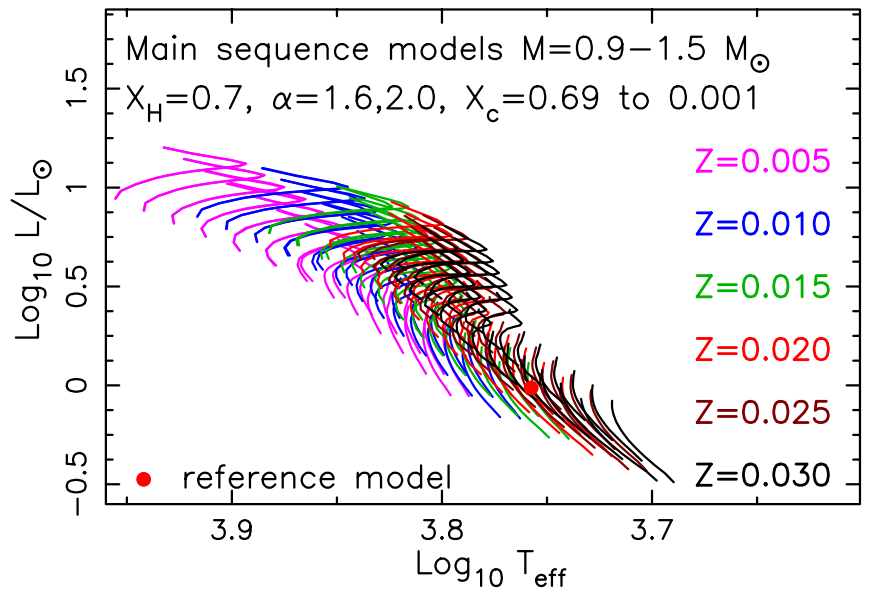

Fig. 7. HR diagram of the main sequence stellar models used in the analysis. The reference model, indicated by the large dot, has $M_{\mathrm{o}}=$ $1 M_{\odot}, R=1.004 R_{\odot}, Z=0.020, \alpha=1.6$ and $\Delta_{\mathrm{o}}=135.8 \mu \mathrm{Hz}$.

One can ask what global constraints should one impose. The two obvious global constraints are the mass $M$ and luminosity $L$, since these are essentially determined solely by the inner structure of the star. The luminosity $L$ can be estimated from parallax and either magnitude or bolometric flux. The mass $M$ may be determined dynamically for stars in binary systems (e.g. $\alpha$ Cen A\&B), or from spectroscopically determined surface gravity $g$, and surface radius $R$ estimated either from interferometry or from $L, T_{\text {eff }}$, the resulting value of $M$ being independent of the structure of the outer layers although dependent on atmospheric modelling. The contributions of the outer layers to $R$ are much smaller than their contribution to $\Delta$; the layers above $x_{f}=0.99$ contribute $1 \%$ to the radius but $17 \%$ to the large separation, so one could impose a radius constraint but with an enhanced error estimate to allow for the uncertain contribution of the outer layers.

\section{Mass estimates from the scaling relation}

As mentioned in the introduction the average large separation is often used to estimate stellar mass using the scaling relation

$$
\frac{M}{M_{\odot}}=\lambda\left(\frac{\Delta}{\Delta_{\odot}}\right)^{2}\left(\frac{R}{R_{\odot}}\right)^{3}, \quad \lambda=\frac{f}{f_{\odot}}
$$

with $\lambda$ set equal to 1 , whereas it actually depends on the values of the dimensionless parameters $f$ which are determined by the composition, evolutionary stage, physics of convection, mixing, diffusion, equation of state ..., so using this relation with $\lambda=1$ is likely to give incorrect estimates of the mass.

We examine this by using stellar models, comparing the masses of the models $M$ with the values $M_{\Delta}$ obtained using the scaling relation with $\lambda=1$ and $R$ and $\Delta$ of the models. For consistency we take as a reference one of the model set with properties similar to the Sun rather than the Sun itself, replacing solar values in Eq. (13) by values of the reference model.

The model set we used is illustrated in Fig. 7: it has main sequence models with masses 0.9 to $1.5 M_{\odot}$, initial composition $X=0.7$, a range of $Z$ values from 0.005 to 0.030 , two values of the mixing length parameter $\alpha=1.6,2.0$, and at various stages of evolution from the zero age main sequence to the terminal main sequence with central hydrogen abundance $X_{\mathrm{c}}=0.001$. The models were computed using the STAROX

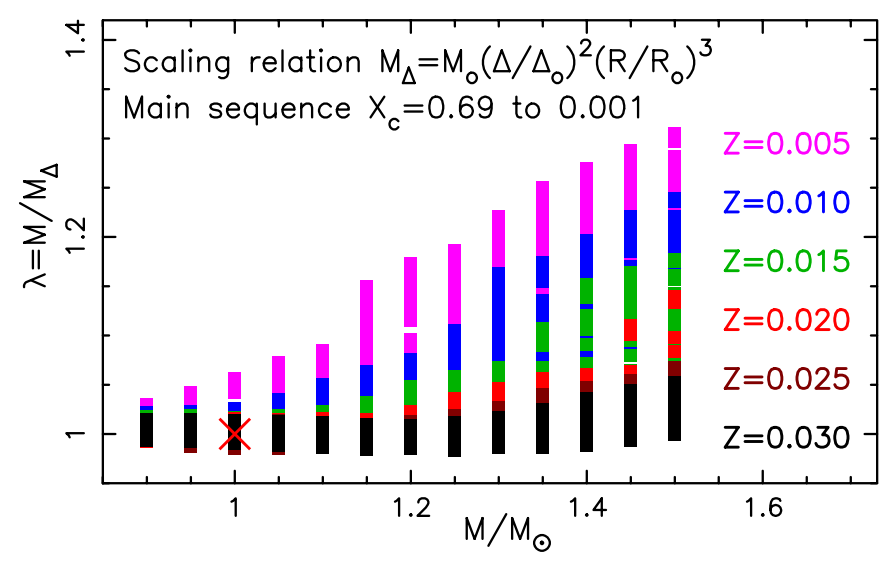

Fig. 8. Comparison of masses of main sequence stellar models with masses from the scaling relation for the set of stellar models shown in Fig. 7. The reference model is indicated by the large cross.

code (Roxburgh 2008b), GN93 relative composition (Grevesse \& Noels 1993), OPAL EOS5 equation of state (Rogers \& Nayfonov 2002), OPAL opacities (Iglesias \& Rogers 1996) supplemented by Wichita low temperature opacities (Ferguson et al. 2005), NACRE nuclear reaction rates (Angulo et al. 1999), no overshooting nor diffusion, and an Eddington atmosphere out to an optical depth of $\tau_{\mathrm{a}}=10^{-3}$.

The frequencies were calculated using the OSCROX code (Roxburgh 2008a), and the average large separations determined from the five $\ell=0$ frequencies centred on a frequency $v_{\max }$ defined as (cf. Brown et al. 1991; Kjeldsen et al. 1995)

$v_{\max }=v_{\odot}\left(\frac{M}{M_{\odot}}\right)\left(\frac{R_{\odot}}{R}\right)^{2}\left(\frac{T_{\odot}}{T_{\mathrm{eff}}}\right)^{0.5}$

with $v_{\odot}=3050 \mu \mathrm{Hz}$ and $T_{\odot}=5777^{\circ} \mathrm{K}$.

Our reference model has a mass $M_{\mathrm{o}}=M_{\odot}, Z=0.02, \alpha=$ $1.6, R_{\mathrm{o}} / R_{\odot}=1.004$ and $\Delta_{\mathrm{o}}=135.8 \mu \mathrm{Hz}$.

The results are displayed Fig. 8, the errors in the scaling relation increase with increasing mass and decreasing metallicity $Z$. The vertical coordinate $\lambda=M / M_{\Delta}$ varies between 0.98 and 1.3. Even larger variations are obtained if one varies other parameters. The cross on the $1 M_{\odot}$ models is the reference model.

In a recent paper Mosser et al. (2013) argued that rather than using the large separations, derived from the observed frequencies, one should use $\Delta_{T}$, the asymptotic value at high frequency defined in terms of the acoustic radius (as in Eq. (3)), and derived by extrapolation from the observed values of $\epsilon_{n \ell}$ defined in Eq. (6). As shown by Hekker et al. (2013) this is not so straightforward, but even if this were possible, it does not solve the problem of the variation of the factor $\lambda$ with dimensionless structure since the acoustic radius is likewise dependent on the factors $f$.

This is illustrated in Fig. 9, where we compare mass estimates from the scaling relation with $\lambda=1$ for the same set of models as in Fig. 8, but now using model values of $\Delta_{T}$ and the value $\Delta_{\text {To }}=138.9 \mu \mathrm{Hz}$ from the reference model. The results are almost identical to those in Fig. 8, demonstrating that there is no improvement in the accuracy of the scaling relation by using the asymptotic large separation $\Delta_{T}=1 /(2 T)$.

\section{Conclusions}

The average large separation, and the acoustic radius, are sensitive to the detailed structure of the outer layers of a star and 


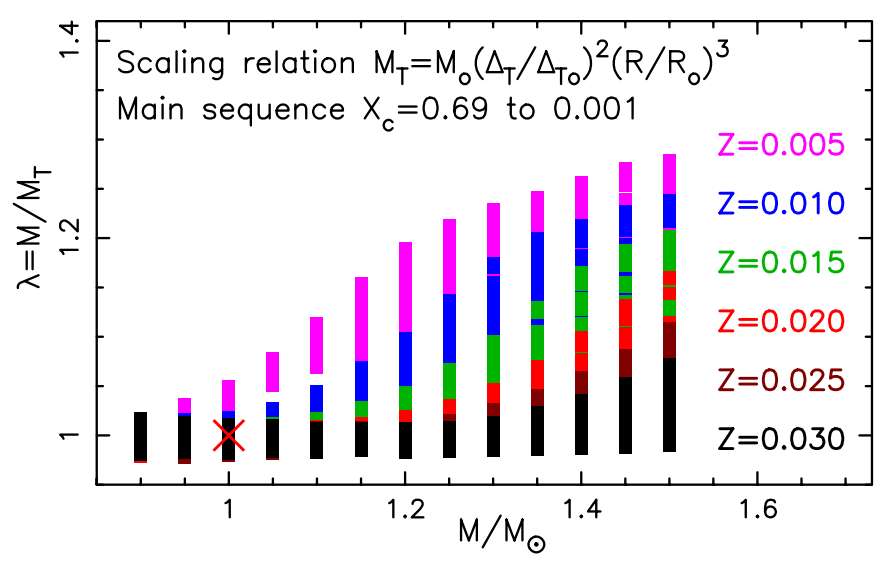

Fig. 9. Same as in Fig. 8 except for using the large separation $\Delta_{T}=$ $1 /(2 T)$ where $T$ is the acoustic radius of the models. The reference model has $\Delta_{\mathrm{To}}=138.9 \mu \mathrm{Hz}$.

therefore should not be used as constraints on surface layer independent model fitting. The luminosity is independent of the outer layers of the star, as is the mass if determined from binary motion or from surface gravity $g$ and surface radius, and both can be used as constraints on surface independent model fitting.

Additional constraints on the models such as the radius, effective temperature, composition and large separation, are not independent of the structure of the outer layers and are subject to the uncertainties discussed above.

Estimates of the stellar mass from the scaling relation are subject to quite large errors due to the different dimensionless structure of stars, and there is no improvement to be gained by using estimates of the asymptotic large separation $\Delta_{T}$ derived from the observed frequencies by extrapolation; $\Delta_{T}=1 /(2 T)$, where $T$ is the acoustic radius, depends on the dimensionless structure of the star in essentially the same way as does the observed average large separation.

Acknowledgements. The author gratefully acknowledges support from the Leverhulme Foundation under grant EM-2012-035/4.

\section{References}

Angulo, C., \& the NACRE consortium. 1999, Nucl. Phys. A, 656, 3 Brown, T. M., Gilliland, R. L., Noyes, R. W., \& Ramsey, L. W. 1991, ApJ, 368, 599

Creevey, O. L., Thevenin, F., Basu, S., et al. 2013, MNRAS, 431, 2419

Christensen-Dalsgaard, J., Dappen, W., \& Lebreton, Y. 1988, Nature, 336, 634

Dziembowski, W. A., Paterno, L., \& Ventura, R. 1988, A\&A, 200, 213

Ferguson, J. W., Alexander, D. R., Allard, D. T., et al. 2005, ApJ, 623, 585

Grevesse, N., \& Noels, A. 1993, In Origin and Evolution of the Elements, eds. N. Prantzos, \& E. Vangioni-Flam (Cambridge Univ. Press), 15

Hekker, S., Elsworth, Y., Basu, S., et al. 2013, MNRAS, 434, 1668

Huber, D., Bedding, T. R., Stello, D., et al. 2011, ApJ, 743, 143

Iglesias, C. A., \& Rogers, F. J. 1996, ApJ, 464, 943

Kjeldsen, H., \& Bedding, T. R. 1995, A\&A, 293, 87

Kjeldsen, H., Bedding, T., \& Christensen-Dalsgaard, J. 2008. ApJ, 683, L175

Mosser, B., Michel, E., Belkacem, K., et al. 2013, A\&A, 550, A126

Rogers, F. J., \& Nayfonov, A. 2002, ApJ, 576, 1064

Roxburgh, I. W. 2006, available at http://www.maths.qmul.ac.uk/ iwr/ oscodes/L1modes.pdf

Roxburgh, I. W. 2008a, Ap\&SS, 316, 141

Roxburgh, I. W. 2008b, Ap\&SS, 316, 75

Roxburgh, I. W. 2009, A\&A, 493, 185

Roxburgh, I. W., \& Vorontsov, S. V. 2000, MNRAS, 317, 141

Roxburgh, I. W., \& Vorontsov, S. V. 2003, A\&A, 411, 215

Roxburgh, I. W., \& Vorontsov, S. V. 2006, MNRAS, 369, 1491

Roxburgh, I. W., \& Vorontsov, S. V. 2013, A\&A, 560, A2

Takata, M. 2005, PASJ, 57, 375

Tassoul, M. 1980, ApJS, 43, 469

Vandakurov, Yu V. 1967, AZh, 44, 786 